\title{
Nasal potential difference measurements in patients with atypical cystic fibrosis
}

\author{
M. Wilschanski*, H. Famini*, N. Strauss-Liviatan", J. Rivlin ${ }^{\star}, H . B^{*}{ }^{+}, H$. Bibi $^{\S}$, L. Bentur $^{f}$,

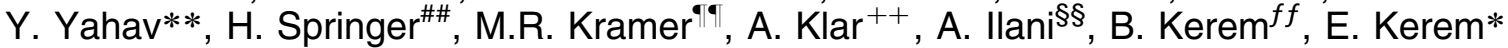

Nasal potential difference measurements in patients with atypical cystic fibrosis. $M$. Wilschanski, H. Famini, N. Strauss-Liviatan, J. Rivlin, H. Blau, H. Bibi, L. Bentur, Y. Yahav, H. Springer, M.R. Kramer, A. Klar, A. Ilani, B. Kerem, E. Kerem. (CERS Journals Ltd 2001.

ABSTRACT: The diagnosis of cystic fibrosis (CF) is based on characteristic clinical and laboratory findings. However, a subgroup of patients present with an atypical phenotype that comprises partial CF phenotype, borderline sweat tests and one or even no common cystic fibrosis transmembrane conductance regulator (CFTR) mutations. The aim of this study was to evaluate the role of nasal potential difference (PD) measurements in the diagnosis of CF patients with an atypical presentation and in a population of patients suspected to have $\mathrm{CF}$.

Nasal PD was measured in 162 patients from four different groups: patients with classical CF $(n=31)$, atypical phenotype $(n=11)$, controls $(n=50)$, and patients with questionable $\mathrm{CF}(\mathrm{n}=\mathbf{7 0})$. The parameter, or combination of nasal PD parameters was calculated in order to best discriminate all CF patients (including atypical CF) from the non-CF group.

The patients with atypical CF disease had intermediate values of PD measurements between the $\mathrm{CF}$ and non-CF groups. The best discriminate model that assigned all atypical $C F$ patients as $C F$ used: $\mathrm{e}^{\text {(response to chloride-free and isoproterenol/response to amiloride) }}$ with a cut-off $>0.70$ to predict a $C F$ diagnosis. When this model was applied to the group of 70 patients with questionable $C F, 24$ patients had abnormal PD similar to the atypical CF group. These patients had higher levels of sweat chloride concentration and increased rate of CFTR mutations.

Nasal potential difference is useful in diagnosis of patients with atypical cystic fibrosis. Taking into account both the sodium and chloride transport elements of the potential difference allows for better differentiation between atypical cystic fibrosis and noncystic fibrosis patients. This calculation may assist in the diagnostic work-up of patients whose diagnosis is questionable.

Eur Respir J 2001; 17: 1208-1215.
*Dept of Pediatrics, Cystic Fibrosis Center, Shaare Zedek Medical Center, Hebrew University Medical School,

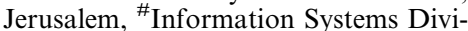
sion, Hadassah Hospital, Jerusalem, Cystic Fibrosis Center, Carmel Medical Center, Haifa, ${ }^{+}$Graub Cystic Fibrosis Center, Schneider Children's Hospital, Petach Tiqva, ${ }^{\S}$ Pediatric Pulmonology Unit, Barzilai Medical Center, Ashkelon, ${ }^{\text {Cystic Fibrosis }}$ Center, Rambam Medical Center, Haifa, **Cystic Fibrosis Center, Sheba Medical Center, Tel Hashomer,

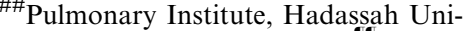
versity Hospital, Jerusalem, "Pulmonary Institute, Rabin Medical Center, Petach Tiqva, ${ }^{++}$Dept of Pediatrics, Bikur Cholim Hospital, Jerusalem, ${ }^{\$ \$}$ Dept of Neurophysiology, Hebrew University and $f f$ Dept of Genetics, Life Sciences Institute, Hebrew University, Jerusalem, Israel.

Correspondence: E. Kerem, Dept of Pediatric Respiratory Medicine and Cystic Fibrosis Center, Shaare Zedek Medical Center, Jerusalem 91031, Israel.

Fax: 97226522176

Keywords: Atypical phenotype cystic fibrosis genotype-phenotype

nasal potential difference

Received: October 252000

Accepted after revision March 62001

This study was supported, in part, by grants from the Chief Scientist of Israeli Health Ministry, The Israeli CF Foundation, and from the Joint Hebrew University - Shaare Zedek Research Fund, The Mirsky Foundation and Balint Charitable Trust
The diagnosis of cystic fibrosis $(\mathrm{CF})$ is made by the presence of typical clinical findings and/or a family history of $\mathrm{CF}$, together with abnormal sweat chloride values and/or the finding of two cystic fibrosis transmembrane conductance regulator (CFTR) mutations $[1-3]$. However, an emerging number of patients present with an atypical phenotype of the disease. These patients may express only partial features of $\mathrm{CF}$ while essential characteristics of the disease may be absent. Such patients may suffer from respiratory disease with sufficient pancreatic function to maintain normal fat absorption, and/or male fertility, and may have normal or intermediate range sweat chloride levels [4-6]. According to the consensus statement, by Rosenstein and CutTing [3], for diagnosis of CF these patients may be diagnosed as CF by the presence of two identified CFTR mutations. Another group of patients may present with phenotypes similar to $\mathrm{CF}$ 
but in only one organ system. These phenotypes may include nasal polyposis [7, 8], chronic sinusitis [9], recurrent pancreatitis $[10,11]$, male infertility due to congenital bilateral aplasia of the vas deferens (CBAVD) [12], allergic bronchopulmonary aspergillosis [13] or unexplained chronic lung disease with no other signs of CF [14-16]. These patients have an increased incidence of CFTR mutations on one CFTR allele. The association between the clinical phenotype and CFTR dysfunction in these patients is unclear.

So far, > 900 CFTR mutations have been identified (L.C. Tsui, CFTR mutations consortium, Hospital for Sick Children, Toronto, Ontario, Canada, personal communication), however, in most populations there are still many $\mathrm{CF}$ chromosomes with unidentified CFTR mutations. In addition, a significant number of patients carry mutations that are uncommon in their population. Patients with the questionable or atypical phenotype tend to carry rare CFTR mutations, mutations that have not yet been identified, or mutations in promotor regions or introns. The search for uncommon or unidentified mutations is complex, time-consuming and expensive. Therefore, it would be difficult to use mutation analysis to diagnose patients with atypical disease.

The basic defect of $\mathrm{CF}$ is due to a dysfunction of chloride ion flux across secretory epithelial cells. In normal epithelia, chloride ions enter the cell across the basolateral membrane and exit the apical surface down an electrochemical gradient via chloride channels. Regulation of these channels is by phosphorylation in response to an increase in intracellular cyclic adenosine monophosphate (cAMP) concentration. Sodium ions enter the apical surface of the cell from the lumen via amiloride-sensitive sodium channels, thus creating a voltage or potential difference (PD) across the apical surface of the cell. KNOwLEs et al. [17] developed a technique to measure the PD across the nasal epithelium, as well as its response to various stimuli. They demonstrated that compared to a control population, in CF basal PD is increased, inhibition with amiloride is exaggerated and there is no significant change in PD after perfusion with chloridefree and isoproterenol solutions. According to the consensus statement, by Rosenstein and CutTing [3], for the diagnosis of $\mathrm{CF}$, nasal $\mathrm{PD}$ may assist in the diagnosis or exclusion of CF. However, it has not been systematically evaluated in patients with atypical CF.

The aim of this study was to evaluate the role of nasal PD measurements in the diagnosis of CF cases with atypical presentation. The results of nasal PD measurements in patients with atypical $\mathrm{CF}$ were compared to those of patients with classical $\mathrm{CF}$ presentation and to normal controls. The results were then applied to a population of patients suspected of having $\mathrm{CF}$.

\section{Methods}

\section{Study subjects}

The present study included 162 patients in four different groups. 1) A control group which comprised normal individuals or patients with non-CF lung disease, including five patients with primary ciliary dyskinesia, immune deficiency and chronic lung disease associated with autoimmune disorders $(n=50$, 19 males; age $30 \pm 5$ yrs mean $\pm S D$ ). 2) A group of $C F$ patients with typical disease $(\mathrm{n}=31,19$ males; age $18 \pm 8$ yrs). CF was diagnosed by typical gastrointestinal and respiratory presentation together with elevated sweat chloride levels (performed in all the study patients according to the GIBSON and CoOKE methodology [18]). 3) Patients with atypical CF $(\mathrm{n}=11$, 9 males; age $26 \pm 12 \mathrm{yrs})$. These patients have partial or mild $\mathrm{CF}$ presentation characterized by sinopulmonary disease and pancreatic sufficiency together with CBAVD, or late diagnosis with milder phenotype. Table 1 shows the clinical characteristics of these patients. In all patients, CF diagnosis was made by elevated sweat chloride levels and/or by the presence of two CFTR mutations when sweat chloride levels were normal or borderline. The mutations carried by these patients have previously been associated with atypical or variable disease presentation [4, 5, 19-21]. 4) Patients with clinical presentation suspected to be $\mathrm{CF}(\mathrm{n}=70,42$ males; age $17 \pm 12$ yrs). These patients were referred for nasal PD measurements from various clinics in Israel. They had at least one of the following clinical features that raised the possibility of $\mathrm{CF}$, but they lacked the

Table 1. - Clinical data of patients with atypical cystic fibrosis disease

\begin{tabular}{|c|c|c|c|c|c|}
\hline Age yrs & Sex & Genotype & Sweat chloride $\mathrm{mmol} \cdot \mathrm{L}^{-1}$ & PS/PI & FEV $1 \%$ pred \\
\hline 22 & M & $3849+10 \mathrm{kbC} \rightarrow \mathrm{T} / 405+1 \mathrm{G} \rightarrow \mathrm{A}$ & 112 & PS & 60 \\
\hline 21 & M & $3849+10 \mathrm{kbC} \rightarrow \mathrm{T} / 405+1 \mathrm{G} \rightarrow \mathrm{A}$ & 70 & PS & 43 \\
\hline 19 & $\mathrm{M}$ & $\mathrm{G} 85 \mathrm{E} / 5 \mathrm{~T}$ & 57 & PS & 72 \\
\hline 15 & M & $5 \mathrm{~T} / ?$ & 110 & PI & 100 \\
\hline 16 & M & $3849+10 \mathrm{kbC} \rightarrow \mathrm{T} / \Delta \mathrm{F} 508$ & 67 & PS & 69 \\
\hline 39 & M & $\mathrm{D} 1152 \mathrm{H} / \mathrm{D} 1152 \mathrm{H}$ & 15 & PS & 100 \\
\hline 52 & M & ?/? & 112 & PI & 35 \\
\hline 38 & $\mathrm{~F}$ & $3849+10 \mathrm{kbC} \rightarrow \mathrm{T} / \mathrm{W} 1282 \mathrm{X}$ & 100 & PS & 44 \\
\hline 23 & $\mathrm{~F}$ & $\Delta \mathrm{F} 508 / ?$ & 80 & PS & 83 \\
\hline 28 & $\mathrm{~F}$ & $3849+10 \mathrm{kbC} \rightarrow \mathrm{T} / \Delta \mathrm{F} 508$ & 80 & PS & 88 \\
\hline 16 & M & $3849+10 \mathrm{kbC} \rightarrow \mathrm{T} / \mathrm{W} 1282 \mathrm{X}$ & 67 & PS & 95 \\
\hline
\end{tabular}

FEV1: forced expiratory volume in one second; M: male; F: female; PS: pancreatic sufficiency; PI: pancreatic insufficiency; ?: unknown. 
criteria required for $\mathrm{CF}$ diagnosis. Fifty-nine patients had sinopulmonary disease including one or more of the following: chronic severe asthma resistant to conventional therapy, recurrent pulmonary infections, bronchiectasis, chronic severe pansinusitis, and nasal polyposis. Four patients had azoospermia or oligospermia. Respiratory disease was evaluated by the presence of clinical symptoms, chest radiograph interpretation, pulmonary function tests and sputum cultures. Primary ciliary dyskinesia was excluded by the absence of ciliary ultrastructural abnormalities. Eleven patients suffered from gastrointestinal manifestations including pancreatic insufficiency; three patients had recurrent pancreatitis, and one patient was asymptomatic and was identified during a family screening as homozygous for the 5T allele. The mean \pm SD sweat chloride levels in this group were $44 \pm 19 \mathrm{mmol} \cdot \mathrm{L}^{-1}\left(\right.$ range $\left.15-83 \mathrm{mmol} \cdot \mathrm{L}^{-1}\right)$.

All patients were tested for the presence of all known CFTR mutations in Israel [22] including the 5T allele [19].

\section{Nasal potential difference measurements}

Transepithelial nasal PD was determined by measuring the PD between a fluid-filled exploring bridge on the nasal mucosa and a reference bridge (21-gauge needle filled with Ringers solution in 4\% agar) inserted into the subcutaneous space of the forearm. Both bridges were linked by calomel electrodes to a highimpedance, low-resistance buffer amplifier. Using direct vision with an otoscope, the exploring catheter was advanced through the inferior meatus of both nostrils and PD was recorded at various sites. After consistent baseline PD measurements had been obtained, the effect of amiloride $\left(1 \times 10^{-4} \mathrm{M}\right)$ superfusion was evaluated. The resultant change in PD was recorded and expressed as both an absolute change and as a percentage change from the baseline maximum PD value. In order to study nasal chloride permeability and cAMP activation of chloride permeability, a large chloride chemical gradient across the apical membrane was generated by superfusion of the nasal mucosa with a chloride-free solution containing $1 \times 10^{-4} \mathrm{~mol} \cdot \mathrm{L}^{-1}$ amiloride at a rate of $5 \mathrm{~mL} \cdot \mathrm{min}^{-1}$. Following this, the mucosa was perfused with the same solution with the addition of isoproterenol $\left(1 \times 10^{-5} \mathrm{~mol} \cdot \mathrm{L}^{-1}\right)$. The change in voltage response over the final 6 min served as an index of epithelial chloride transport.

\section{Deoxyribonucleic acid sequence determination and mutation analysis}

Deoxyribonucleic acid (DNA) sequences spanning individual exons of the $\mathrm{CF}$ gene were amplified by polymerase chain reaction (PCR) with oligonucleotide primers located in the respective flanking introns of the $\mathrm{CF}$ gene $[23,24]$. The amplified genomic DNA fragments eluted from $5 \%$ polyacrylamide gels were extracted with chloroform and subjected to the dideoxy-chain termination sequencing method as described, using the US Biochemicals Sequenase kit (Cleveland, OH, USA) with either one of the PCR primers or internal oligonucleotides as sequencing primers. Following the identification of a specific mutation in an individual, the entire CF population studied was detected for this mutation using previously described methods [22]. Analysis of the polythymidine tract at the branch/acceptor site of exon 9 was performed as previously described [19].

\section{Statistical analysis}

Mean values and standard deviations (SDs) were computed for all nasal PD parameters: basal PD, response to amiloride perfusion in $\mathrm{mV}$ and per cent change, and response to chloride-free and isoproterenol solutions, for each of the $\mathrm{CF}$, atypical $\mathrm{CF}$, and non-CF control groups. Comparison between the three patient groups was performed separately on each parameter, using multiple comparison methods with a significance level of 0.05 . For multiple comparisons, analysis of variance (ANOVA) was used with the Bonferroni and Dunnetts procedures for the post hoc tests, as appropriate.

The results of nasal PD measurements included several parameters. Discriminant analysis was used in order to explore which parameter, or combination of parameters, can best discriminate all $\mathrm{CF}$ patients (including atypical $\mathrm{CF}$ ) from the non-CF group. The parameters used were basal PD, response to amiloride perfusion in $\mathrm{mV}$ and per cent change, and response to chloride-free and isoproterenol solutions. Various combinations and functional forms of the relevant variables were tested to achieve the best separation between the groups by the discriminant function. Fisher's linear discriminant function was applied to suggest a cut-off point between $\mathrm{CF}$ and non-CF patients.

The estimated model was then used to assign the 70 suspected CF patients into two groups: CF or non$\mathrm{CF}$. The suspected CF group, assigned by the model as $\mathrm{CF}$, was then compared to each of the two groups of $\mathrm{CF}$ patients, $\mathrm{CF}$ and atypical $\mathrm{CF}$ using DunNeTTs [25] test for each of the PD parameters. Comparison of values of sweat chloride and forced expiratory volume in one second (FEV1) between the suspected $\mathrm{CF}$ patients who were assigned $\mathrm{CF}$ and those who were assigned as non-CF, was performed using an unpaired t-test, separately on each of these two parameters. The Chi-squared test was performed to test the association between genotypes and the predicted groups. Data are presented as mean \pm SD unless otherwise stated.

The Human Ethics Committee of The Israeli Ministry of Health approved the study and informed written consent was obtained from all the patients or parents.

\section{Results}

All patients with classical CF had typically abnormal nasal PD measurements compared to controls 
Table 2. - Nasal potential difference (PD) results of cystic fibrosis (CF) patients, control, patients with atypical CF disease, and patients with questionable CF disease

\begin{tabular}{lccccc}
\hline & Patients $\mathrm{n}$ & Basal PD mV & $\Delta$ Amiloride $\mathrm{mV}$ & $\Delta$ Amiloride $\%$ & $\Delta$ Iso + chloride-free $\mathrm{mV}$ \\
\hline Non-CF & 50 & $-16 \pm 5$ & $10 \pm 4$ & $61 \pm 18$ & $-12 \pm 7$ \\
CF & 31 & $-49 \pm 13^{* * *}$ & $35 \pm 11^{* * *}$ & $72 \pm 13^{*}$ & $3 \pm 6^{* * *}$ \\
Atypical CF & 11 & $-35 \pm 16^{* * *}$ & $25 \pm 10^{* * *}$ & $73 \pm 18^{*}$ & $0 \pm 2^{* * *}$ \\
QCF & 70 & $-23 \pm 10$ & $13 \pm 8$ & $54 \pm 19$ & $-6 \pm 5$ \\
QCF-CF & 24 & $-30 \pm 13$ & $18 \pm 12$ & $60 \pm 20$ & $-1 \pm 3$ \\
QCF-non-CF & 46 & $-19 \pm 5^{* *}$ & $10 \pm 4^{* *}$ & $51 \pm 18$ & $-9 \pm 4^{* *}$ \\
\hline
\end{tabular}

Data are presented as mean + SD. Iso: isoproterenol; CF: cystic fibrosis; QCF: questionable cystic fibrosis; QCF-CF: questionable $\mathrm{CF}$ with abnormal PD; QCF-non-CF: questionable $\mathrm{CF}$ with normal $\mathrm{PD} .{ }^{*} \mathrm{p}<0.05$, for the comparison with non$\mathrm{CF} ;{ }^{* *} \mathrm{p}<0.01$, for the comparison between QCF-non-CF and QCF-CF; ***p $<0.001$, for the comparison with non-CF.

(table 2). CF patients had increased sodium transport, as demonstrated by elevated negative basal PD $(\mathrm{p}<0.001)$ (fig. 1a), exaggerated response to amiloride perfusion in absolute values $(\mathrm{p}<0.001)$ (fig. $1 \mathrm{~b})$ and per cent change from basal PD $(\mathrm{p}=0.016)$, and no chloride transport, as demonstrated by lack of response to perfusion with isoproterenol and chloridefree solution $(\mathrm{p}<0.001)$ (fig. 1c).

The patients with atypical $\mathrm{CF}$ disease, as a group, had intermediate PD measurements between the $\mathrm{CF}$ and non-CF groups (table 2). As shown in figures 1a, $1 \mathrm{~b}$ and $1 \mathrm{c}$, compared to the control group, patients with atypical CF had significantly higher negative basal PD $(\mathrm{p}<0.001)$, increased response to amiloride perfusion in absolute values $(\mathrm{p}<0.001)$, higher per cent change from baseline $(p=0.02)$, and no response to isoproterenol and chloride-free solution $(\mathrm{p}<0.001)$. Similar to the classical CF group, patients with atypical CF had absent chloride transport (no response to perfusion with isoproterenol and chloridefree solution) $(\mathrm{p}=0.42)$, and increased response to amiloride perfusion in per cent change from baseline $(\mathrm{p}=1.0)$. However, the patients with atypical $\mathrm{CF}$ had lower basal PD $(\mathrm{p}<0.001)$ and decreased amiloride response in absolute values $(\mathrm{p}=0.001)$, when compared to classical CF.

Therefore, the discriminate analysis was used to explore which parameter, or combination of parameters best classify the atypical $\mathrm{CF}$ patients as $\mathrm{CF}$ and discriminate them from the non-CF group. The best
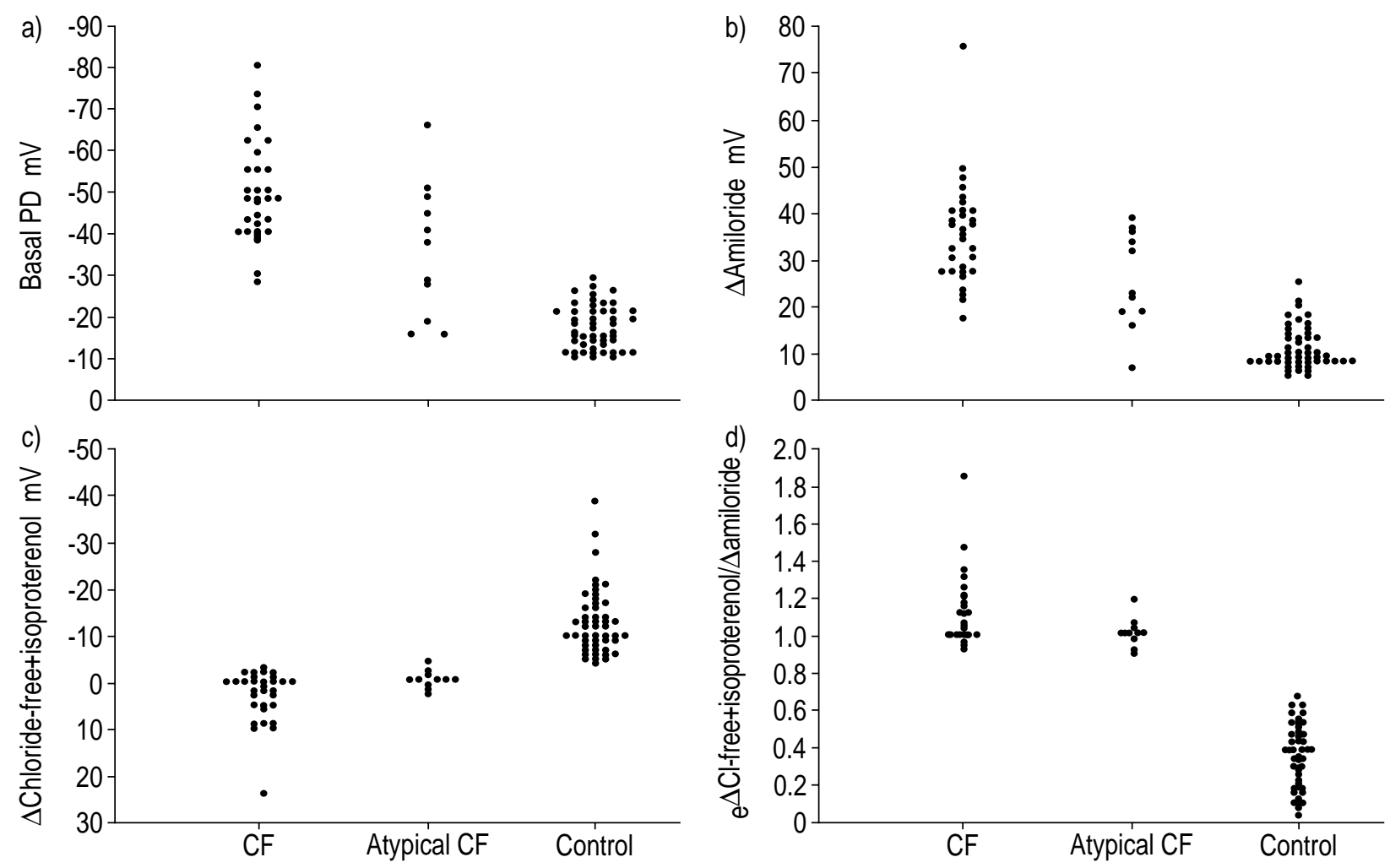

Fig. 1. - a) Basal potential difference (PD) in cystic fibrosis $(C F)(n=31)$, atypical $C F(n=11)$ and control patients $(n=50)$. Note that some atypical CF patients had normal basal PD. b) The change in PD following amiloride perfusion in $C F(n=31)$, atypical $C F(n=11)$ and control patients $(n=50)$. c) The change in PD following perfusion of chloride $(\mathrm{Cl})$-free and isoproterenol solution in $\mathrm{CF}(\mathrm{n}=31)$, atypical CF $(n=11)$ and control patients $(n=50)$. d) The exponent of the ratio: response to chloride-free+isoproterenol/response to amiloride in $C F(n=31)$, atypical $C F(n=11)$ and control patients $(n=50)$. 
discriminate model is one using the exponent of the ratio:response to isoproterenol and chloride-free/ response to amiloride ( $\mathrm{e}^{\text {(response to chloride-free and }}$ isoproterenol/response to amiloride) as the best discriminative variable between the groups. Using Fisher's linear discriminate function, the cut-off is given by the inequality ( $\mathrm{e}^{\text {(response to chloride-free and isoproterenol/response }}$ to amiloride) $>0.70$, which is equivalent to the actual ratio, without the exponent, of the sum of the response to isoproterenol and chloride-free/response to amiloride $>-0.35$ to be a CF patient (fig. 1d).

The group of 70 patients with questionable $\mathrm{CF}$ was then studied. The basal PD in this group was $-23 \pm 10 \mathrm{mV}$, the change in PD following amiloride perfusion was $13 \pm 8.5 \mathrm{mV}$, and the repolarization following superperfusion with chloride-free solution and isoproterenol was $-6.3 \pm 5.3 \mathrm{mV}$. However, when the statistical model was applied to this group of patients, two distinct patterns of the PD results emerged, enabling the segregation of these patients into two groups: 1) 46 patients with normal measurements similar to the non-CF controls, questionable $\mathrm{CF}$ with normal PD (QCF-non-CF); and 2) 24 patients with abnormal measurements, questionable CF with abnormal PD (QCF-CF). As shown in table 2, there were significant differences in all the PD parameters between the two groups including basal PD, response to amiloride superperfusion, response to chloride-free solution and isoproterenol superperfusion. However, none of these parameters discriminated clearly between the two groups (figs. $2 \mathrm{a}$ and $b)$.

The group of QCF-CF patients was compared to each of the two groups of $\mathrm{CF}$ patients: classical $\mathrm{CF}$ and atypical CF. The QCF-CF group had measurements similar to the atypical $\mathrm{CF}$ group in basal $\mathrm{PD}$ $(p=0.46)$, in response to amiloride perfusion $(p=0.18)$ and in response to chloride-free solution and isoproterenol superperfusion $(\mathrm{p}=0.77)$, but still significantly different in all these parameters from the classical $\mathrm{CF}$ patients $(\mathrm{p}<0.001,<0.001$ and $=0.002$, respectively). This showed that the QCF-CF group was similar to the atypical CF group.

Clinical parameters and genotype analysis of the two groups of patients are shown in table 3 . There was no difference in pulmonary function between both groups. However, the group of patients with QCF-CF had a lower mean age and significantly higher mean sweat chloride levels compared to that of the QCF-non-CF group.

Genotype analysis for the known mutations in the Israeli population [21] revealed that none of the patients from the QCF-non-CF group carried two CFTR mutations or the 5T allele on both chromosomes, apart from one patient homozygous for 5T, compared to five patients $(22 \%)$ in the QCF-CF group, $\mathrm{p}<0.001$ (table 3). The frequency of the $5 \mathrm{~T}$ allele in the QCF-CF group was $17.4 \%$, significantly higher than the $6 \%$ frequency in the general population [19] $(\mathrm{p}=0.04)$, but not significantly higher than the $10.5 \%$ frequency in the QCF-non-CF. Interestingly, the frequency of $\Delta \mathrm{F} 508$ among the QCF-non-CF was $8.5 \%$ which was significantly higher than the
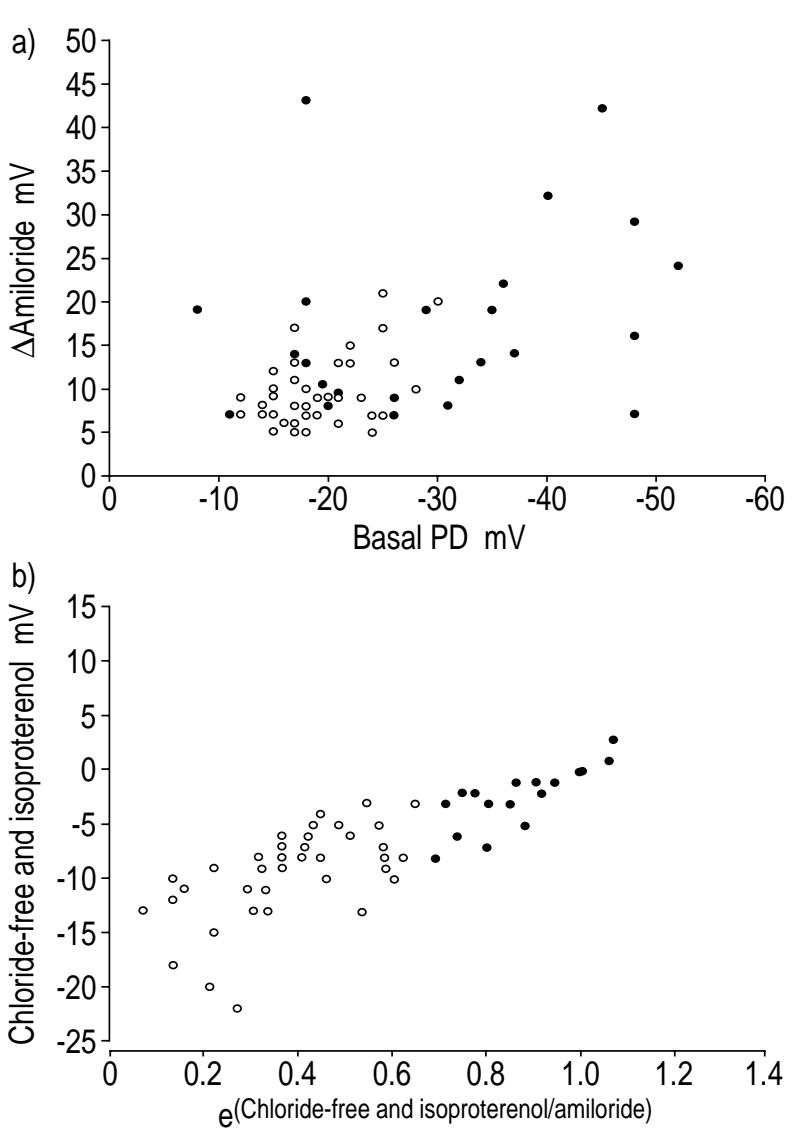

Fig. 2. - a) The change in potential difference (PD) following amiloride solution plotted against basal PD in questionable $\mathrm{CF}$ $(O ; n=24)$ and questionable non-CF patients $(\bigcirc ; n=46)$. b) The change in PD following chloride-free and isoproterenol solution plotted against the exponent of the ratio:response to isoproterenol and chloride-free/response to amiloride in questionable $\mathrm{CF}(\mathbf{O}$; $\mathrm{n}=24)$ and questionable non-CF patients $(\bigcirc ; \mathrm{n}=46)$.

frequency of $1.4 \%$ in the Israeli population [26] $(\mathrm{p}<0.01)$.

\section{Discussion}

Nasal PD measurement is a well-established electrophysiological tool for the diagnosis of CF and for the monitoring of treatment protocols both in animal models $[27,28]$ and CF patients [29-35]. The present study shows that this test is abnormal in patients with atypical CF disease. However, some patients with atypical CF may have borderline or even normal basal PD and amiloride response, but characteristically abnormal chloride conductance. It is therefore imperative not to rely on the basal PD only and to complete the PD measurement with chloride-free and isoproterenol perfusion before a diagnosis of $\mathrm{CF}$ is ruled out. The chloride secretory response has high sensitivity and specificity for normal versus $\mathrm{CF}$ or atypical $\mathrm{CF}$. In a similar manner to the atypical $\mathrm{CF}$ patients in the present study, the patients in a study by WALKER et al. [36] carried mutations associated with milder pulmonary disease and had typical abnormal PD measurements. Likewise, PRADAL et al. [37] 
Table 3. - Clinical parameters of patients referred with questionable cystic Fibrosis (CF)

\begin{tabular}{lccc}
\hline & QCF-CF & QCF-non-CF & p-value \\
\hline Subjects $n$ & 24 & 46 & \\
Age yrs & $12.17 \pm 10.49$ & $19.13 \pm 11.9$ & 0.013 \\
Sweat chloride $\mathrm{mmol} \cdot \mathrm{L}^{-1}$ & $56 \pm 17$ & $39 \pm 18$ & $<0.001$ \\
FEV1 \% pred & $83.6 \pm 19.7$ & $83.7 \pm 32.9$ & $\mathrm{NS}$ \\
Positive sputum clutures & 4 & $5 \mathrm{~T} / 5 \mathrm{~T}(1)$ & $\mathrm{NS}$ \\
CFTR mutations & $\mathrm{W} 1282 \mathrm{X} / 5 \mathrm{~T}(2)$ & $\Delta \mathrm{F} 508(3)$ & $<0.001^{+}$ \\
& $\mathrm{W} 1282 \mathrm{X} / 3849+10 \mathrm{kbC}->\mathrm{T}(1)$ & $5 \mathrm{~T}(3)$ & $0.04^{+}$ \\
& $\Delta \mathrm{F} 508 / 5 \mathrm{~T}(1)$ & & \\
Male infertility & $\mathrm{D} 1152 \mathrm{H} / 5 \mathrm{~T}(1)$ & 3 & $\mathrm{NS}$ \\
Pancreatic insufficiency & $\Delta \mathrm{F} 508(1)$ & 1 & $\mathrm{NS}$ \\
\hline
\end{tabular}

Data are preserted as mean $\pm \mathrm{SD}$. \#: Pseudomonas(5); Hemophilus influenza(5). Moraxella(2); Streptococcus(1); ': for comparison of the number of patients carrying two CFTR mutations in both groups; ${ }^{+}$: for comparison of the frequency of the 5T allele in the QCF-CF group to that in the general population. QCF-CF: questionable CF with abnormal potential difference. QCF-non-CF: questionable CF with normal potential difference; FEV1: forced expiratory volume in one second; CFTR: cystic fibrosis transmembrane conductance regulator; NS: nonsignificant.

measured nasal PD in males with CBAVD and showed that a subgroup of patients had normal basal PD, but abnormal chloride transport values. In another study, this group measured the basal PD in a group of CF patients with borderline sweat chloride levels and found that some of them had normal basal PD measurements [38]. DANNER et al. [39] compared nasal PD between controls, proven $\mathrm{CF}$ patients and a group of patients with disseminated bronchiectasis and found that the latter group had normal PD.

Although patients with atypical CF may have abnormal chloride transport, the cut-off where a diagnosis of $\mathrm{CF}$ can be made is not always clear. According to the model in the present study, the technique of nasal PD might best define atypical CF patients as $\mathrm{CF}$ by applying a statistical model using the ratio of the response to isoproterenol and chloride-free/response to amiloride. This model classified all atypical $\mathrm{CF}$ patients as $\mathrm{CF}$ and was found to be the best to clearly discriminate them from the non$\mathrm{CF}$ subjects. It is interesting to note that this function considers both chloride transport (expressed by response to isoproterenol and chloride-free solutions) and sodium transport (expressed by response to amiloride). An actual value of $>-0.35$ (equivalent to the exponential ratio of $>0.7$ ) was found to predict a $\mathrm{CF}$ diagnosis. However, further studies in other laboratories and on a larger number of patients are required to confirm the present findings.

Patients presenting with disease resembling the atypical CF phenotype may share signs and symptoms with other diseases, such as severe asthma, immune deficiency, ciliary dyskinesia, postinfectious or idiopathic segmental bronchiectasis or idiopathic pancreatitis. Furthermore, in these patients, genetic analysis usually reveals that they do not carry two common CFTR mutations. Pursuing a thorough CFTR mutation search in cases where CF diagnosis is uncertain is prohibitive. It is therefore, difficult to know if all these patients have CF. The results of the present study in a large group of patients in whom diagnosis of $\mathrm{CF}$ cannot be established or ruled-out by sweat test or mutation analysis, show that a subgroup of patients had nasal PD studies with abnormal response to chloride-free and isoproterenol solutions and intermediate values of basal PD and amiloride depolarization. These PD measurements were similar to the group of proven atypical CF patients. These patients with abnormal PD also had higher sweat chloride values and a significantly higher incidence of patients carrying two CFTR mutations.

Thus, it is likely that these patients have atypical CF disease. However, further studies including search for the rare CFTR mutations, whole genome scan, or CFTR functional studies will be required to associate their disease with CFTR dysfunction.

Wilson et al. [40] studied 11 patients with questionable diagnosis of $\mathrm{CF}$ who underwent nasal PD as part of their diagnostic evaluation. Two of their patients had an abnormal PD and subsequently both were found to carry the 5T allele on one chromosome. In the present study, of the 24 patients with QCF who had an abnormal PD, five patients were found to carry two CFTR mutations, four of them the 5T allele on one chromosome. The 5T allele has been described as a variant with partial penetrance causing disease with an extremely variable clinical presentation [19]. Recently, it has been shown that two patients with respiratory symptoms and carrying the $5 \mathrm{~T}$ allele had abnormal chloride transport [41]. Thus, the PD measurements in the present study patients confirm previous clinical and molecular observations that the $5 \mathrm{~T}$ allele might be associated with atypical $\mathrm{CF}$ disease [19, 23].

An interesting finding was the increased frequency of the $\Delta F 508$ mutation among patients with questionable CF and normal nasal PD. It is possible that the disease in some of the patients with normal PD might be associated with CFTR dysfunction, which together with environmental or other genetic factors (modifier genes) lead to the development of pulmonary disease. Numerous studies have reported increased frequency of the $\Delta \mathrm{F} 508$ mutation among patients with chronic bronchopulmonary disease 
including chronic bronchitis, bronchiectasis, allergic bronchopulmonary aspergillosis $[12-16,42]$ and among patients with asthma [43, 44]. All these observations suggest that heterozygosity to the $\Delta \mathrm{F} 508$ mutation may predispose to recurrent or chronic pulmonary diseases.

In conclusion, nasal potential difference measurements are useful in the diagnosis of patients with atypical cystic fibrosis disease. It is imperative not to rely on the basal potential difference and to complete the potential difference measurements with chloridefree and isoproterenol superperfusion before a diagnosis of cystic fibrosis is ruled-out. The cut-off point between normal and abnormal chloride transport should be determined in each laboratory. Patients with questionable cystic fibrosis and with potential difference measurements showing abnormal chloride and sodium transport are likely to have atypical cystic fibrosis. Thus, further studies to determine the frequency of cystic fibrosis transmembrane conductance regulator mutations in this patient population are needed.

\section{References}

1. Rosenstein BJ, Zeitlin PL. Cystic fibrosis. Lancet 1988; 351: 277-281.

2. Stern RC. Current concepts: the diagnosis of cystic fibrosis. N Engl J Med 1997; 336: 487-491.

3. Rosenstein BJ, Cutting GR. The diagnosis of cystic fibrosis: a consensus statement. J Pediatr 1998; 132: $589-595$.

4. Stewart B, Zabner J, Shuber AP, Welsh MJ, McCray B Jr. Normal sweat chloride values do not exclude the diagnosis of cystic fibrosis. Am J Respir Crit Care Med 1995; 151: 899-903.

5. Highsmith WE, Lauraneli H, Burch MS, et al. A novel mutation in the cystic fibrosis gene in patients with pulmonary disease but normal sweat chloride concentrations. N Engl J Med 1994; 331: 974-980.

6. Augarten A, Kerem B, Yahav Y, et al. Mild cystic fibrosis and normal or borderline sweat test in patients with the $3849+10 \mathrm{~kb} \mathrm{C}->\mathrm{T}$ mutation. Lancet 1993; 342: $25-26$.

7. Irving RM, McMahon R, Clark R, Jones NS. CFTR mutations in severe nasal polypsis. Clin Otolaryngol 1997; 22: 519-521.

8. Burger J, Macek M Jr, Stuhrmann M, Reis A, Krawczak M, Schmidtke J. Genetic influences in the formation of nasal polyps. Lancet 1997; 337: 974.

9. Wiatrak BJ, Meyer CM, Cotton RT. Cystic fibrosis presenting with sinus disease in children. Am J Dis Child 1993; 147: $258-260$.

10. Cohn JA, Friedman KJ, Noone PG, Knowles MR, Silverman LM, Jowell PS. Relation between mutations of the cystic fibrosis gene and idiopathic pancreatitis. $N$ Engl J Med 1998; 339: 653-658.

11. Sharer N, Schwarz M, Malone G, et al. Mutations of the cystic fibrosis gene in patients with chronic pancreatitis. N Engl J Med 1998; 339: 645-652.

12. Chillon M, Casals T, Mercier B, et al. Mutations in the cystic fibrosis gene in patients with congenital bilateral absence of the vas deferens. $N$ Engl J Med 1995; 332: $1475-1480$.

13. Miller PW, Hamosh A, Macek M Jr, et al. Cystic fibrosis transmembrane conductance regulator (CFTR) gene mutations in allergic bronchopulmonary aspergillosis. Am J Hum Genet 1995; 59: 45-51.

14. Giorodon E, Cazeneuve C, Lebargy F, et al. CFTR mutations in adults with disseminated bronchiectasis. Eur J Hum Genet 1997; 5: 149-155.

15. Dumur V, Lafitte JJ, Gervais R, et al. Abnormal distribution of cystic fibrosis $\Delta \mathrm{F} 508$ allele in adults with chronic bronchial hypersecretion. Lancet 1990; 335: 1340.

16. Gervais R, Lafitte JJ, Dumur V, et al. Sweat chloride and $\Delta \mathrm{F} 508$ mutation in chronic bronchitis or bronchiectasis. Lancet 1993; 342: 997.

17. Knowles MR, Paradiso AM, Boucher RC. In vivo nasal potential difference: techniques and protocols for assessing efficacy of gene transfer in cystic fibrosis. Hum Gene Ther 1993; 6: 445-455.

18. Gibson LE, Cooke RE. A test for concentration of electroytes in sweat in cystic fibrosis of the pancreas utilizing pilocarpine by iontophoresis. Pediatrics 1959; 23: $545-549$.

19. Kerem E, Rave-Harel N, Augarten A, et al. A cystic fibrosis transmembrane conductance regulator splice variant with partial penetrance associated with variable cystic fibrosis presentations. Am J Respir Crit Care Med 1997; 155: $1914-1920$.

20. Feldmann D, Rochemaure J, Plouvier E, Magnier C, Chauve C, Aymard P. Mild course of cystic fibrosis in an adult with the D1152H mutation. Clin Chem 1995; 41: 1675 .

21. Kerem E, Nissim-Rafinia M, Argaman Z, et al. A missense cystic fibrosis transmembrane conductance regulator mutation with variable phenotype. Pediatrics 1997; 100: E5.

22. Kerem E, Kalman YM, Yahav Y, et al. Highly variable incidence of cystic fibrosis and different mutation distribution among different Jewish ethnic groups in Israel. Hum Gene 1995; 96: 193-197.

23. Rave-Harel N, Kerem E, Nissim-Rafinia M, et al. The molecular basis of partial penetrance of splice mutations in cystic fibrosis. Am J Hum Genet 1997; 60: $87-$ 94.

24. Shoshani T, Kerem E, Szeinberg A, et al. Similar levels of mRNA from the $\mathrm{W} 1282 \mathrm{X}$ and the $\Delta \mathrm{F} 508$ cystic fibrosis alleles in nasal epithelial cells. $J$ Clin Invest 1994; 93: $1502-1507$.

25. Dawson-Saunders B, Trapp RG. In: Basic and Clinical Biostatistics. Norwalk, Appleton and Lange, 1990; pp. $8-12$.

26. Abeliovich D, Lavon IP, Lerer I, et al. Screening for five mutations detects $97 \%$ of cystic fibrosis chromosomes and predicts a carrier frequency of 1:29 in the Jewish Ashkenazi population. Am J Hum Genet 1992; 51: $951-956$.

27. Wilschanski M, Rozmahel R, Beharry S, et al. In vivo measurements of ion transport in long-living mice. Biochem Biophys Res Commun 1996; 219: 753 - 759.

28. Ramjeesingh M, Huan LJ, Li C, et al. Assessment of the efficacy of in vivo CFTR protein replacement therapy in CF mice. Hum Gene Ther 1998; 9: 521 - 528.

29. Knowles MR, Hohneker KW, Zhou Z, et al. A controlled study of adenoviral-vector-mediated gene transfer in the nasal epithelium of patients with cystic fibrosis. N Engl J Med 1998; 333: 823-831.

30. Rubenstein RC, Zeitlin PL. A pilot clinical trial of oral sodium-4-phenylbutyrate in $\Delta \mathrm{F} 508$ homozygous 
cystic fibrosis patients. Am J Respir Crit Care Med 1998; 157: 484-490.

31. Zabner J, Ramsey BW, Meeker DP, et al. Repeat adminstration of an adenovirus vector encoding cystic fibrosis transmembrane regulator to the nasal epithelium of patients with cystic fibrosis. J Clin Invest 1996; 97: $1504-1511$.

32. Zabner J, Chen SH, Meeker D, et al. Comparison of DNA-lipid complexes and DNA alone for gene transfer to cystic fibrosis airway epithelia in vivo. J Clin Invest 1998; 100: 1529-1537.

33. Arispe N, Ma J, Jacobson KA, Pollard HB. Direct activation of cystic fibrosis transmembrane conductance regulator (CFTR) by the alkylxanthine 8-cyclopentyl-1 3-dipropylxanthine (CPX) and 1,3diallylcyclohexa manthine (DAX). J Biol Chem 1998; 273: $5727-5734$

34. Kelley TJ, Thomas K, Milram LJ, Drumm MK. In vivo activation of the cystic fibrosis transmembrane conductance regulator mutant $\Delta \mathrm{F} 508$ in murine nasal epithelium. Proc Natl Acad Sci USA 1998; 94: 2604-2608.

35. Alton EW, Stern M, Farley R, et al. Cationic lipidmediated CFTR gene transfer to the lungs and nose of patients with cystic fibrosis: a double-blind placebo controlled trial. Lancet 1999; 353: 947-954.

36. Walker LC, Venglarik CJ, Gervais A, et al. Relationship between airway ion transport and a mild pulmonary disease mutation in CFTR. Am J Respir Crit Care Med 1997; 155: $1684-1689$.
37. Pradal U, Castellani C, Delmarco A, Mastella G. Nasal potential difference in congenital bilateral absence of the vas deferens. Am J Respir Crit Care Med 1998; 158: 896-901.

38. Delmarco A, Pradal U, Cabrini G, Bonizzato A, Mastella G. Nasal potential difference in cystic fibrosis patients presenting borderline sweat test. Eur Respir $J$ 1997; 10: $1145-1149$.

39. Danner I, Boisseau P, Chailleux E, Escande D. Respiratory epithelial ion transport in patients with disseminated bronchiectasis. Eur Respir J 1999; 13: $1276-1280$.

40. Wilson D, Ellis L, Zielenski J, et al. Uncertainty in the diagnosis of cystic fibrosis: possible role of in vivo nasal potential difference measurements. Pediatr 1998; 132: $596-599$.

41. Noone PG, Pue CA, Zhou Z, et al. Lung disease associated with the IVS8 5T allele of the CFTR gene. Am J Respir Crit Care Med 2000; 162: 1919-1924.

42. Puechal X, Fajac I, Bienvenu $\mathrm{T}$, et al. Increased frequency of cystic fibrosis $\Delta \mathrm{F} 508$ mutation in bronchiectasis associated with rheumatoid arthritis. Eur Respir J 1999; 13: 1281 - 1287.

43. Lazaro C, de Cid R, Sunyer J, et al. Missense mutations in the cystic fibrosis gene in adult patients with asthma. Hum Mutat 1999; 14: 510-519.

44. Dahl M, Tybjaerg-Hansen A, Lange P, Nordestgaard BG. Delta F508 heterozygosity in cystic fibrosis and susceptibility to asthma. Lancet 1998; 351: $1911-1913$. 